\title{
Lumbopelvic Stabilization Exercises and McKenzie Method in Low Back Pain Due to Disc Protrusion: A Blind Randomized Clinical Trial
}

\author{
M. B. Dohnert ${ }^{1}$, C. Schwanck Borges ${ }^{2}$, A. Steffen Evaldt ${ }^{2}$, C. de Jesus Francisco ${ }^{2}$, \\ L. da Silva Dias'2, B. Chuaste Flores², G. Maciel Bello², R. Dimer da Luz², R. Boff Daitx² \\ 1 Research, Teaching and Extension Laboratory in Orthopedic Trauma Physiotherapy (LAPEFITO), Gurupi \\ University (UnirG), Gurupi, Torres, Brazil \\ 2 Lutheran University of Brazil, Torres, Rio Grande do Sul, Brazil
}

\section{CORRESPONDING AUTHOR:}

Marcelo Baptiista Dohnert

Research, Teaching and Extension

Laboratory in Orthopedic Trauma

Physiotherapy (LAPEFITO)

Department of Physiotherapy

University of Gurupi - UnirG

355 Joaquim Batista de Oliveira Street

77.403-170 Vila Alagoana

Gurupi/TO, Brazil

E-mail: mdohnert@hotmail.com

DOI:

10.32098/mltj.04.2020.22

LEVEL OF EVIDENCE: $1 B$

\begin{abstract}
SUMMARY
Introduction. Disc Protrusion (DP) is a degenerative spinal disorder. Lumbar intervertebral disc protrusion is one of the most common orthopedic injuries, leading to low back pain that radiates to the lower limbs. Physical exercise is the main element in the conservative treatment of low back pain.

Objective. Comparing the effects of the McKenzie method and core stabilization exercises on the rehabilitation of patients with lumbar DP.

Methods. We allocated sixty-nine patients with low back pain due to disc protrusion to the Core Group (CG), the McKenzie Group (MG), or the Core + McKenzie Group (CMG). All groups underwent three interventions per week for four weeks, totaling 12 sessions. We assessed pain intensity, hamstring flexibility, functional capacity, muscle strength, and lumbopelvic stability.

Results. All intervention groups significantly reduced pain intensity and functional disability, besides improving posterior muscle chain flexibility, quality of life, trunk muscle strength, and lumbopelvic stability $(\mathrm{p}<0.05)$. For functional capacity, assessed through the SF-26 Questionnaire, as well as for posture holding time in the lumbopelvic stability tests, the CG and $\mathrm{CMG}$ significantly improved the results compared to the MG $(\mathrm{p}<0.05)$.

Conclusions. The results suggest that both exercise methods were efficient in reducing pain and improving function in patients with low back pain due to disc protrusion. However, the groups that used stabilization exercises showed better results in activating lumbopelvic stabilizing muscles.
\end{abstract}

\section{KEY WORDS}

Low back pain; intervertebral disc displacement; physical therapy modalities; exercise therapy; spine osteoarthritis.

\section{INTRODUCTION}

Low back pain is a major health problem, with enormous economic and social costs (1). Disc Protrusion (DP) is a degenerative spinal disorder (1). The degenerated disc has focal or localized abnormalities on the disc margin $(2,3)$. This disorder relates to age, gender, prolonged workload, trauma, and pregnancy (4). Lumbar intervertebral disc protrusion is one of the most common orthopedic conditions, producing, in many cases, low back pain that radiates to the lower limbs
$(5,6)$. Lumbar radiculopathy may be the result of a lumbar disc protrusion or hernia that can irritate a sacral plexus trunk, leading to intraneural inflammation (6). A protruding or herniated degenerated disc can cause paresthesia and weakness in the lower limbs, in addition to pain (6). In the worst cases, lumbar radiculopathy can be chronic, resulting in pain, disability, and substantial burden in the long run (6). This condition leads to health-related socioeconomic consequences that directly affect well-being and quality of life $(5,7)$. 
Degenerated protruding discs and narrow spinal canals are common conditions in the elderly (8). However, most subjects adapt perfectly well to the degenerative findings of imaging tests, keeping in shape, and avoiding poor posture (8). Physical activity is the main element in the prevention and treatment of low back pain (9). It does not require expensive materials, therefore providing an accessible intervention in which the patient directly controls pain (9).

Spinal stabilization and the McKenzie approach are two different interventions for the treatment and prevention of low back pain (9). The core stabilization program emphasizes the training of specific deep or local stabilizing muscles of the spine, keeping the lumbar region in a neutral position during occupational and daily life activities (10). The McKenzie method, also called Mechanical Diagnosis and Therapy (MDT) (8), emphasizes an examination/ treatment that incorporates repeated spinal movements $(10,11)$. The patient's response to these repeated movements determines a classification and a preferred direction for therapeutic exercise (11-14). Postural correction and maintenance of normal lordosis are an integral part of the McKenzie program, and treatment programs generally emphasize the performance of repeated lumbar movements for the effective management of specific syndromes (13). This method considers the patient's symptomatic and mechanical response to repeated movements and sustained postures for evaluation and treatment $(8,14)$. Therefore, it uses a directional preference to reduce or increase the patient's symptoms $(12,14)$.

The spinal stabilization system, also called core system, is composed of the spinal column, which provides intrinsic stability, muscles that provide dynamic stability, and a neural control unit that evaluates and determines the stability and coordination requirements of the muscle response (8). The central stabilizing muscles, also called core muscles, are categorized, according to their function, as global muscles (rectus abdominis, spinal erectors, and external obliquus abdominis) and local muscles (transversus abdominis and lumbar multifidus) (15). Global muscles participate in trunk movements, while local muscles play an important role in stabilizing the core (15). The transversus abdominis and the lumbar multifidus play important roles in the functional activities of the spine (15). Core stabilization exercises aim to activate central muscles, including the transversus abdominis, lumbar multifidus, and paraspinal, abdominal, diaphragmatic, and pelvic muscles. This provides spinal stability and prevents lumbopelvic injuries by controlling joint tension, strengthening muscles, increasing resistance, and correcting posture $(7,16-20)$.

The present study compares the effects of the McKenzie method and core stabilization exercises, as well as their association, on the rehabilitation of patients with low back pain due to disc protrusion.

\section{METHODS}

Blind randomized clinical trial conducted from August 2017 to June 2019. The study was registered in the Brazilian Clinical Trials Registry (REBEC) under the identification number RBR-5mcvt6. The study was approved by the Committee of Ethics on Human Research of Universidade Luterana do Brasil under the number 2, 152, 592 (21).

\section{Sample calculation}

We used pain intensity, measured by VAS, as the primary outcome of the study. Based on the study by Hasanpour-Dehkordi, Dehghani and Solati (11), we used a study power of $80 \%$, a $95 \%$ Confidence Interval, and a sample size ratio of 1:1:1 (Core; McKenzie; Core + McKenzie) to estimate the number of participants for each intervention group. Believing that losses and refusals would be around $20 \%$, we reached the final number of 23 participants for each study group, totaling 69 participants.

\section{Sample randomization}

After the initial assessment, we randomized the eligible participants by using a list of random numbers provided by the EPI-Info ${ }^{\circledR}$ software. We allocated a total of 69 patients to the following groups: McKenzie Group (MG), which performed only one McKenzie exercise protocol, Core Group (CG), to which we applied lumbopelvic stabilization exercises, and Core + McKenzie Group (CMG), which performed both protocols (figure 1).

\section{Elegibility criteria}

The study included 69 patients of both genders, aged between 18 and 65 years, who had low back pain due to disc protrusion. The participants should have complementary exams (magnetic resonance or computed tomography) showing DP and/or herniated disc, and should not be performing any other type of physiotherapeutic approach for low back pain at the time of the study. All participants signed the Informed Consent Form (ICF).

We excluded from the study patients who had severe spinal pathologies (fractures, tumors, and inflammatory pathologies such as ankylosing spondylitis), who had previously undergone lumbar surgery, patients with severe cardiovascular and cardiorespiratory disease, pregnant women, patients who did not attend three consecutive or intermit- 


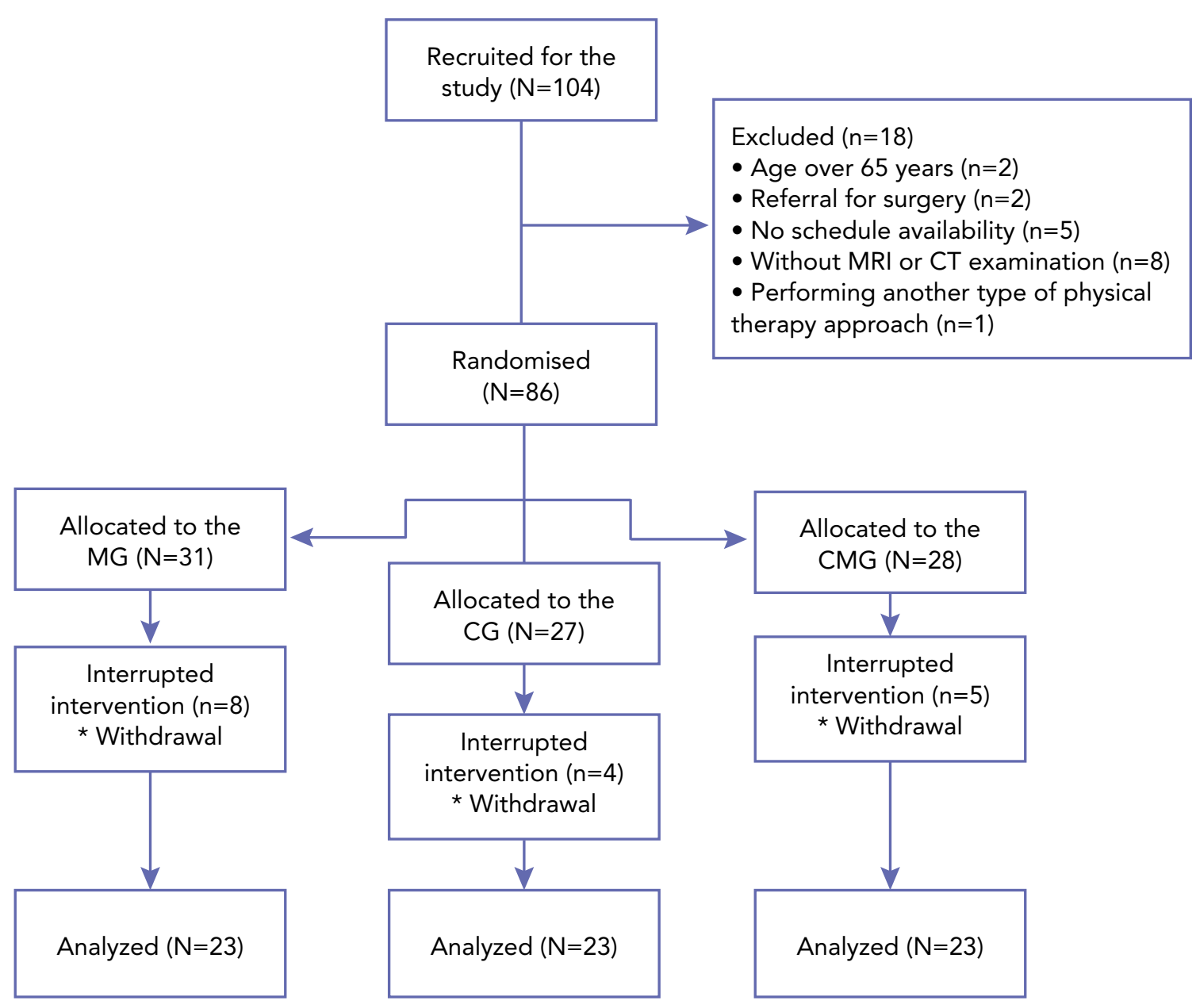

Figure 1. Flowchart - Recruitment and evaluation of patients with disc protrusion.

RM: MRI; CT: Computed Tomography; CG: Core Group; MG: McKenzie Group; CMG: Core + McKenzie Group.

tent consultations, and those that presented some neurological disorder that prevented them from performing the proposed exercises.

\section{Data collection}

The evaluation protocol was conducted by a blind evaluator, that is who did not know which group the participant belonged to. The evaluation took place in two stages of the study: initially - prior to the randomization of the subjects, and at the end of the intervention protocol.

\section{Anthropometric assessment}

We calculated the Body Mass Index (BMI) by measuring body height and weight.

\section{Pain intensity assessment}

We assessed pain intensity by using the Visual Analogue Scale (VAS). In this scale, the evaluator asks the patient about his/her pain level on a scale from zero to ten, where zero means total absence of pain and ten the maximum tolerable pain.

\section{Quality of life assessment}

We assessed quality of life by using the SF-36 Questionnaire. This instrument consists of 11 questions and 36 items that encompass eight domains represented by functional capacity, physical aspects, pain, general health status, vitality, social aspects, emotional aspects, and mental health. The application of this questionnaire takes place through the attribution of a score for each question, later trans- 
formed into a scale from " 0 " to " 100 " per domain, where " 0 " corresponds to the worst quality of life and " 100 " the best quality of life.

\section{Functional capacity assessment}

To quantify disability, we used the Roland-Morris Disability Questionnaire. This instrument consists of 24 items that describe daily tasks that patients have difficulty performing due to low back pain. The questions have a dichotomous answer (yes or no), and the result corresponds to the sum of the yes answers. This result can vary between 0 and 24 , with zero corresponding to a person without complaints, and the maximum value corresponding to a patient with very severe limitations. The greater the number of alternatives filled in, the greater the functional impairment. We instructed the individuals to complete the items that better described them on the day of application.

\section{Hamstring flexibility assessment}

We measured hamstring flexibility by using the Wells bench. For performing this test, the patient seated with legs extended and feet supported on the device. Then, the individual should flex his/her trunk and hips the maximum possible, with hands superimposed and supported on the measuring tape installed on the upper part of the Wells bench. The evaluator asked the patient to stretch his/her hands as far as he/she could reach without bending the knees, thus obtaining the maximum reach point.

\section{Trunk extensor muscle strength assessment}

We assessed trunk extensor muscle strength by using the Crown ${ }^{\circledR}$ dorsal dynamometer. In this test, the patient remained standing, with feet supported and parallel, knees extended, and the spine initially flexed. At the command of the examiner, the patient extended the spine by holding the lever bar with his/her upper limbs for dynamometer measurement.

\section{Lumbopelvic stability assessment}

To analyze lumbopelvic stability, we applied the Single Leg Squat Test (unilateral squat), the Runner Pose Test, the Prone Instability Test, the Right and Left Lateral Bridge Test, and the Sorenson Test (extensor endurance test).

\section{Intervention protocol}

The intervention protocol consisted of three interventions per week for four weeks, with all study groups performing a total of 12 sessions. The service took place individually. Each session lasted between 40 and 60 minutes. Initially, all participants performed a 10-minute stationary bicycle warm-up. Afterwards, we performed the intervention according to the randomized group. The intervention protocol was applied by a researcher previously trained and familiar with the protocol. The interventor controlled during the sessions the technique of performing each exercise, as well as its intensity and duration in both intervention groups.

\section{Intervention protocol for the McKenzie Group}

The intervention protocol for the MG consisted of three sets of exercises with 10 repetitions each, with a 30-second interval between sets. We maintained the exercises for five seconds initially, subsequently maintaining them for 10 seconds according to the patient's performance. We defined the treatment from the result of the evaluation performed prior to the randomization stage, and guided it according to the preferred direction of movement, that is flexion, extension, or lateral displacement of the spine. We applied the McKenzie protocol according to Garcia et al. (13) (table I).

\section{Intervention protocol for the Core Group}

In the CG protocol, we developed the exercises according to the studies of Moon et al. (21), who used eight exercises based on static postures, initially maintained for five seconds. As the program evolved, the degree of difficulty

Table I. McKenzie group exercise protocol.

\begin{tabular}{ll}
\hline Exercise & Number of repetitions \\
\hline Exercise 1. Flexion of the lower limbs on the bench press & 3 sets with 10 repetitions \\
\hline Exercise 2. Bending the trunk while sitting on the chair & 3 sets with 10 repetitions \\
\hline Exercise 3. Standing trunk flexion & 3 sets with 10 repetitions \\
\hline Exercise 4. Prone trunk extension & 3 sets with 10 repetitions \\
\hline Exercise 5. Standing trunk extension & 3 sets with 10 repetitions \\
\hline Exercise 6. Lateralization of the standing pelvis & 3 sets with 10 repetitions for each side \\
\hline Exercise 7. Lateralization of the pelvis against the wall & 3 sets with 10 repetitions for each side \\
\hline
\end{tabular}


in controlling the posture increased, with the posture holding time changing to 10 seconds. Participants of this group repeated each exercise 10 times, with a 30-second interval between them. The researcher commanded continuous contraction of stabilizers ("abdominal press") in all exercises (table II).

Intervention protocol for the Core + McKenzie group In the CMG protocol, patients performed both exercise methods in association. The patients started with McKenzie exercises and performed core stabilization exercises after a five-minute rest according to the defined protocol.

\section{Statistical analysis}

We used the Statistical Package for the Social Sciences (SPSS) version 23.0 for data and statistical analyses. We entered the data twice so as to avoid typing errors, and expressed them as mean and standard deviation. Afterwards, we statistically analyzed the data by parametric tests: Analysis of Variance (ANOVA) for repeated measures within each group from baseline to endpoint, and unpaired Student's t Test for intergroup analysis at each time point. For nonparametric variables, we used the Wilcoxon and Chi-Square Tests, respectively. We established the level of significance of $\mathrm{p}<$ 0.05 for the statistical test.

\section{RESULTS}

The study initially included 104 participants. Of these, 18 were excluded and 86 were randomized to the study groups. Among the randomized participants, 17 discontinued intervention due to withdrawal. Thus, the present study analyzed a total of 69 participants (figure 1). Of these, 25 (36.23\%) were men, with an average age of 46 years. The groups were homogeneous for the variables skin color, occupation, time of pain, and anthropometric aspects (table III).

All intervention groups decreased pain intensity after the intervention protocol, with no differences between them ( $\mathrm{p}$ $<0.001$ ) (figure 2).

Hamstring flexibility increased significantly in all intervention groups, with no differences between them (figure 3).

All intervention groups decreased the Roland-Morris Disability Questionnaire (RMDQ) score $(\mathrm{p}<0.001)$. The scores did not differ between the intervention groups (figure 4).

All study groups also improved quality of life according to the SF-36 Questionnaire, with no differences between them (figure 5). The three intervention groups improved all domains of the questionnaire. However, for the Functional Capacity domain, the CG showed a more significant variation in the score than the MG and CMG $(\mathrm{p}=0.018)$ (table IV).

Regarding isometric trunk muscle strength (MVIC), all groups increased isometric strength after the intervention, with no differences between them (figure 6).

Regarding lumbopelvic stability tests, all study groups significantly improved the scores of the Single Leg Squat Test, Runner Pose Test, and Prone Instability Test $(\mathrm{p}<$ 0.05). Notwithstanding, when analyzing the results between the groups, the CG and CMG scored better than the MG (p $<0.05)$ (table V).

All intervention groups improved the scores of the Lateral Bridge and Sorenson tests after the intervention $(p<0.05)$. The CG and CMG showed a significantly higher variation in posture holding time than the MG in the Left and Right Lateral Bridge Test $(\mathrm{p}=0.009$ and $\mathrm{p}=0.012$, respectively) (table VI).

Table II. Core stabilization Group exercise protocol.

\begin{tabular}{ll}
\hline Exercise & Number of repetitions \\
\hline Exercise 1. Bench press & 10 times from 5 to 10 sec., 10 sec. rest \\
\hline Exercise 2. Bird dog & 10 times from 5 to 10 sec. for each side \\
\hline Exercise 3. Lateral bridge & 10 times from 5 to 10 sec. for each side \\
\hline Exercise 4. Prone bridge & 10 times from 5 to 10 sec., 10 sec. rest \\
\hline Exercise 5. Isometry (lower portion of the rectus abdominis) & 10 times from 5 to 10 sec., 10 sec. rest \\
\hline Exercise 6. Supine bridge with extended lower limbs & 10 times from 5 to 10 sec., 10 sec. rest \\
\hline Exercise 7. Minisquat on the wall with a Swiss ball & 10 times from 5 to 10 sec., 10 sec. rest \\
\hline Exercise 8. Isometry (bench press flexion) & 10 times from 5 to 10 sec., 10 sec. rest
\end{tabular}


Table III. Characterization of the study sample $(n=69)$.

\begin{tabular}{|c|c|c|c|c|}
\hline \multirow[b]{2}{*}{ Variable } & \multicolumn{3}{|c|}{ Intervention group } & \multirow[b]{2}{*}{$\mathbf{p}$} \\
\hline & CG $(n=23)$ & MG $(n=23)$ & CMG $(n=23)$ & \\
\hline Age, years $($ mean \pm SD) & $45.43 \pm 10.40$ & $51.00 \pm 6.81$ & $43.96 \pm 10.56$ & $0.034^{\#}$ \\
\hline Skin color, $\mathrm{n}(\%)$ & & & & $1.000^{\$}$ \\
\hline White & $23(100.0)$ & $23(100.0)$ & $23(100.0)$ & \\
\hline Farmer & $1(4.3)$ & $5(21.7)$ & $6(26.0)$ & \\
\hline Homekeeper & $2(8.7)$ & $7(30.4)$ & $3(13.0)$ & \\
\hline Joiner & $1(4.3)$ & $1(4.3)$ & $0(0.0)$ & \\
\hline Teacher & $1(4.3)$ & $0(0.0)$ & $2(8.7)$ & \\
\hline Time of pain, months (mean \pm SD) & $72.70 \pm 66.44$ & $112.83 \pm 80.88$ & $104.74 \pm 117.63$ & $0.135^{8}$ \\
\hline Directional preference & & & & $0.129^{\$}$ \\
\hline Flexion & $13(56.5)$ & $16(69.6)$ & $10(43.5)$ & \\
\hline Extension & $6(26.1)$ & $7(30.4)$ & $11(47.8)$ & \\
\hline Side slope & $4(17.4)$ & $0(0.0)$ & $2(8.7)$ & \\
\hline Weight, $\mathrm{Kg}($ mean $\pm \mathrm{SD})$ & $77.42 \pm 11.15$ & $76.70 \pm 14.26$ & $72.96 \pm 13.50$ & $0.465^{\#}$ \\
\hline Height, cm (mean \pm SD) & $167.22 \pm 9.47$ & $164.39 \pm 7.52$ & $157.90 \pm 35.37$ & $0.330^{\#}$ \\
\hline $\mathrm{BMI}, \mathrm{Kg} / \mathrm{cm}^{2}($ mean $\pm \mathrm{SD})$ & $27.69 \pm 3.52$ & $28.21 \pm 3.79$ & $26.80 \pm 4.08$ & $0.448^{\#}$ \\
\hline
\end{tabular}

Legend: CG=Core Group; MG=McKenzie Group; CMG=Core + McKenzie Group; Kg: kilogram; cm: centimeters; M: male; F: female.

" One-way ANOVA.

$\$$ Chi-square.

${ }^{\&}$ Kruskal-Wallis test.

\section{DISCUSSION}

This study compares the effectiveness of two exercise programs for low back pain due to DP, also analyzing the association of these two programs. The results do not support the initial hypothesis that the core stabilization exercise program is more effective than the McKenzie exercise program in relieving pain and improving posterior muscle chain flexibility and functional disability scores in individuals with low back pain secondary to disc protrusion/degeneration. Both programs, alone and in association, led to positive results.

The analyses did not reveal statistical differences in pain intensity, isometric trunk extensor strength, and function. These results corroborate those found in other previously published studies $(10,22,24,25)$. However, specific tests for lumbopelvic-hip complex stabilization showed that the groups that performed core stabilization exercises had greater postural control than the group that performed only the McKenzie exercise program.

Spinal stabilization exercises and the McKenzie approach are two different interventions generally used to treat low back pain. The stabilization program applied in this study addressed specific muscle abnormalities associated with low back pain due or related to degenerative discopathy and DP. The program emphasized the training of deep and local spinal stabilizing muscles, keeping the lumbar region in a neutral position $(10,22,23)$. The McKenzie approach emphasizes an examination/treatment that incorporates repeated spinal movements. The patient's response to these repeated movements determines a classification and a preferred direction for therapeutic exercise $(10,22,23)$. O'Sullivan et al. (26) observed that several participants in their study needed four to five weeks to learn how to coactivate Transverse Abdominis (TrA) and multifidus muscles. 


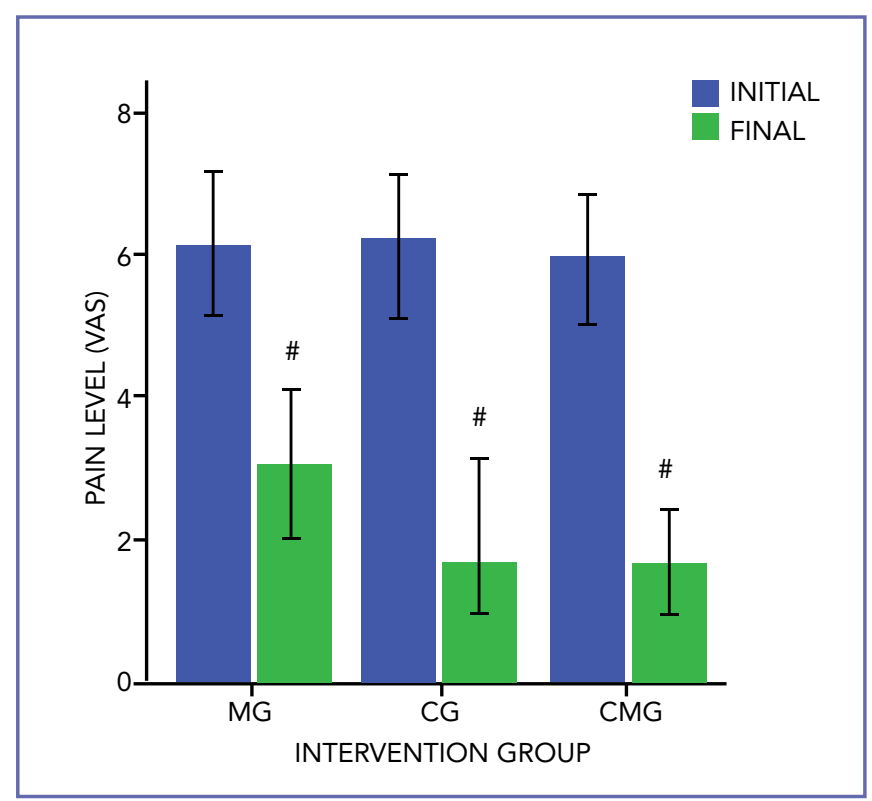

Figure 2. Pain intensity assessment in the study groups before and after intervention.

CG: Core Group; MG: McKenzie Group; CMG: Core + McKenzie Group; VAS: Visual Analogue Scale.

$\# p<0.001$ compared to baseline in the same group. Student's t test.

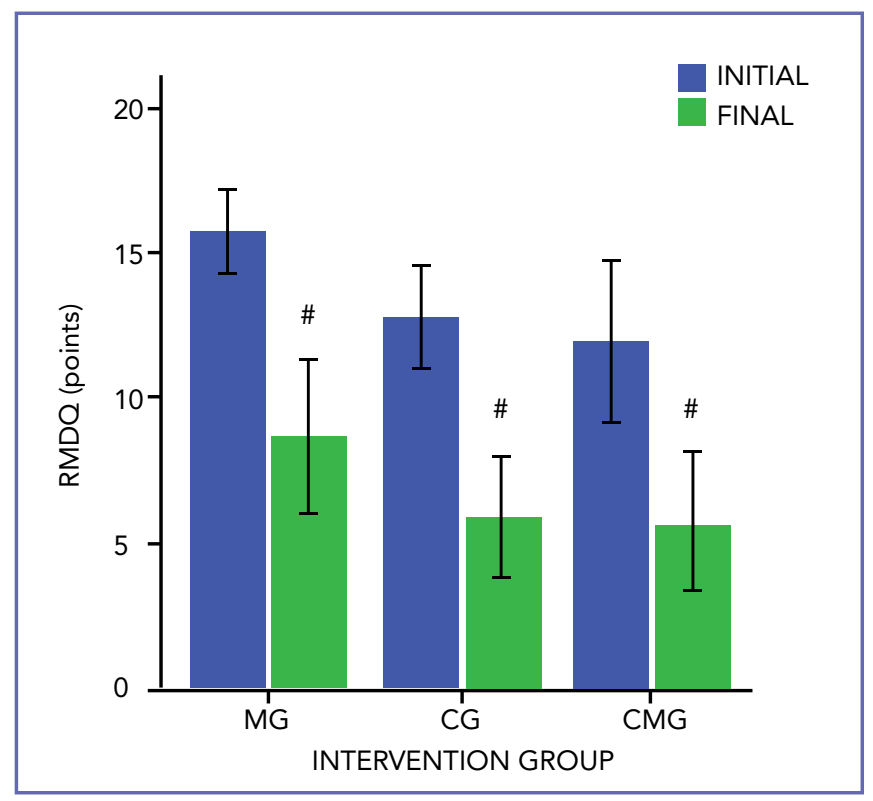

Figure 4. Roland-Morris Disability Questionnaire (RMDQ) score in the study groups before and after intervention.

GC: Core Group; MG: McKenzie Group; CMG: Core + McKenzie Group.

\# $p<0.001$ compared to baseline in the same group. Student's $\mathrm{t}$ test.

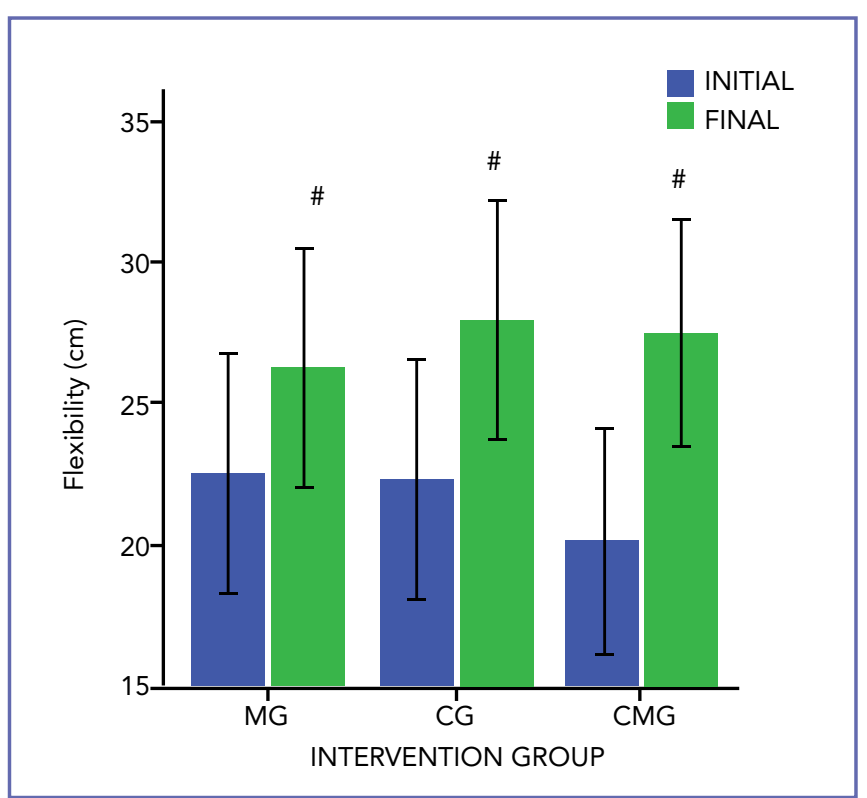

Figure 3. Hamstring flexibility assessment through the Wells bench in the study groups before and after intervention.

GC: Core Group; MG: McKenzie Group; CMG: Core + McKenzie Group.

$\# p<0.001$ compared to baseline in the same group. Student's t test.

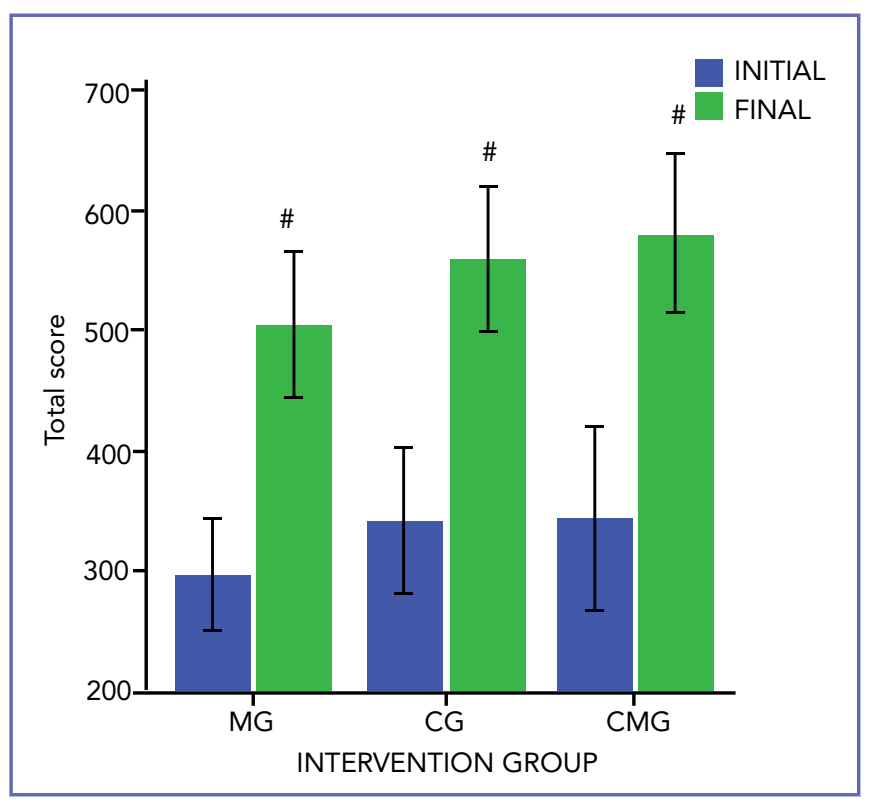

Figure 5. Total SF-36 score in the study groups before and after intervention.

GC: Core Group; MG: McKenzie Group; CMG: Core + McKenzie Group.

$\# p<0.001$ compared to baseline in the same group. Student's t test.

Muscles, Ligaments and Tendons Journal 2020;10 (4) 
Table IV. Evaluation of the SF-36 questionnaire subitems $(n=69)$.

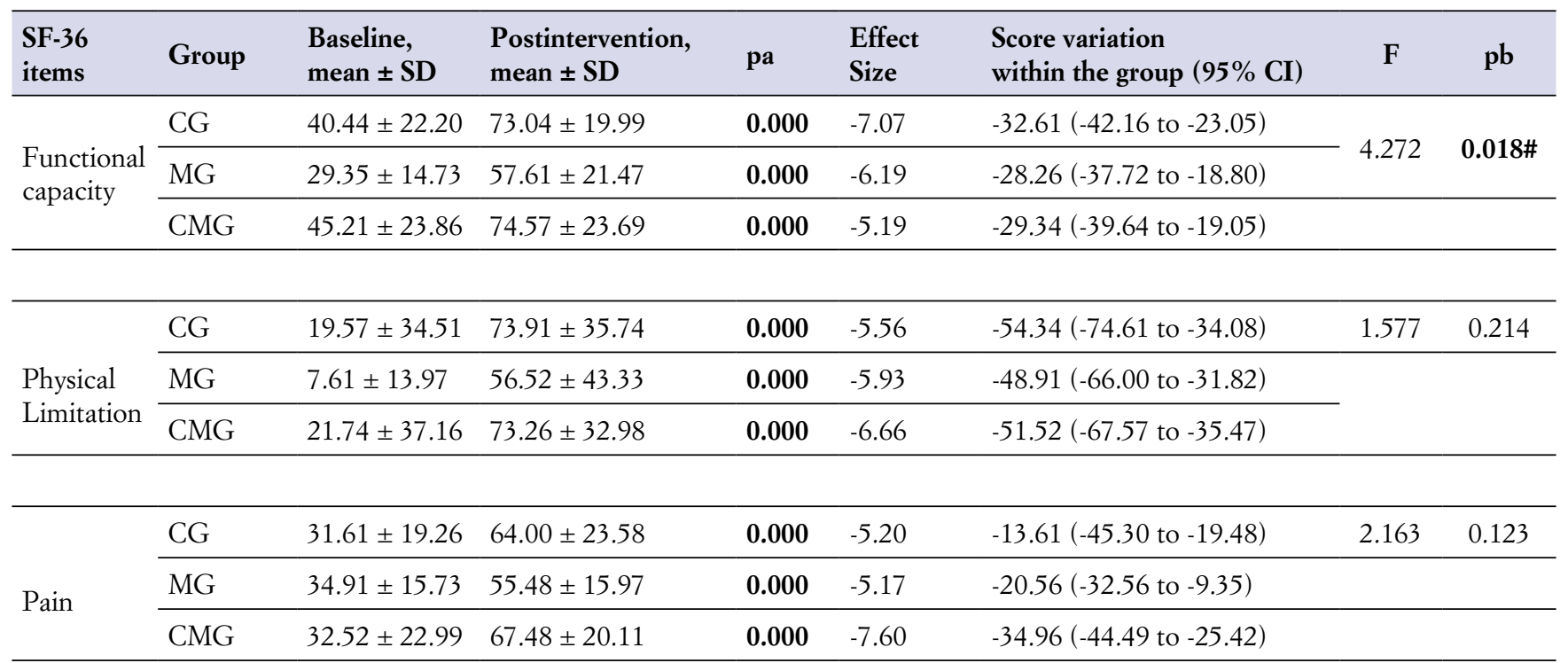

\begin{tabular}{|c|c|c|c|c|c|c|c|c|}
\hline & CG & $53.26 \pm 22.81$ & $66.87 \pm 22.82$ & 0.001 & -3.83 & $-13.61(-20.97$ to -6.24$)$ & 0.800 & 0.454 \\
\hline $\begin{array}{l}\text { Health } \\
\text { Condition }\end{array}$ & $\mathrm{CMG}$ & $46.61 \pm 24.79$ & $64.96 \pm 21.18$ & 0.000 & -4.24 & $-18.35(-27.33$ to -9.37$)$ & & \\
\hline
\end{tabular}

\begin{tabular}{|c|c|c|c|c|c|c|c|c|}
\hline \multirow{2}{*}{ Vitality } & CG & $41.52 \pm 19.51$ & $60.65 \pm 22.92$ & 0.001 & -3.85 & $-19.13(-29.43$ to -8.83$)$ & 2.498 & 0.090 \\
\hline & CMG & $49.57 \pm 25.49$ & $70.35 \pm 19.08$ & 0.000 & -4.66 & $-20.78(-30.04$ to -11.52$)$ & & \\
\hline
\end{tabular}

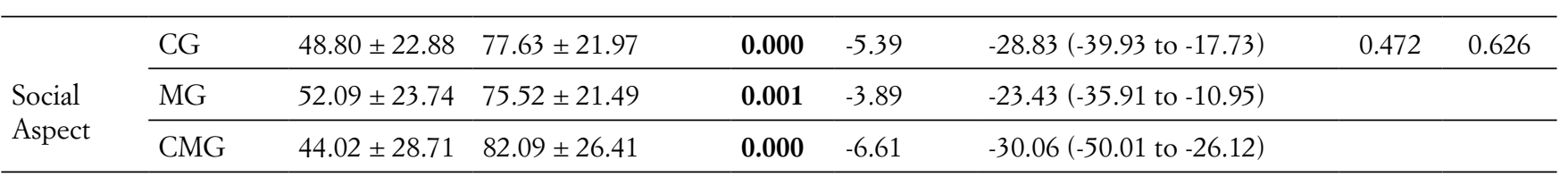

\begin{tabular}{|c|c|c|c|c|c|c|c|c|}
\hline & CG & $52.12 \pm 45.86$ & $76.76 \pm 40.78$ & 0.037 & -2.21 & $-24.66(-47.74$ to -1.57$)$ & 0.073 & 0.930 \\
\hline & CMG & $40.56 \pm 47.09$ & $74.33 \pm 36.00$ & 0.001 & -3.88 & $-33.77(-51.81$ to -15.72$)$ & & \\
\hline
\end{tabular}

\begin{tabular}{|c|c|c|c|c|c|c|c|c|}
\hline & CG & $56.35 \pm 24.39$ & $71.13 \pm 18.84$ & 0.011 & -2.78 & $-14.78(-25.79$ to -3.78$)$ & 0.890 & 0.445 \\
\hline $\begin{array}{l}\text { Mental } \\
\text { Health }\end{array}$ & CMG & $62.78 \pm 22.45$ & $73.39 \pm 21.43$ & 0.003 & -3.36 & $-10.61(-17.15$ to -4.07$)$ & & \\
\hline
\end{tabular}

Legend: CG: Core Group; MG: McKenzie Group; CMG: Core + McKenzie Group; CI: Confidence Interval; SD: Standard Deviation.

Bold values are statistically significant.

a Intragroup Student's t-test.

b One-way ANOVA.

\# Significant difference between the MG, CG, and CMG. 


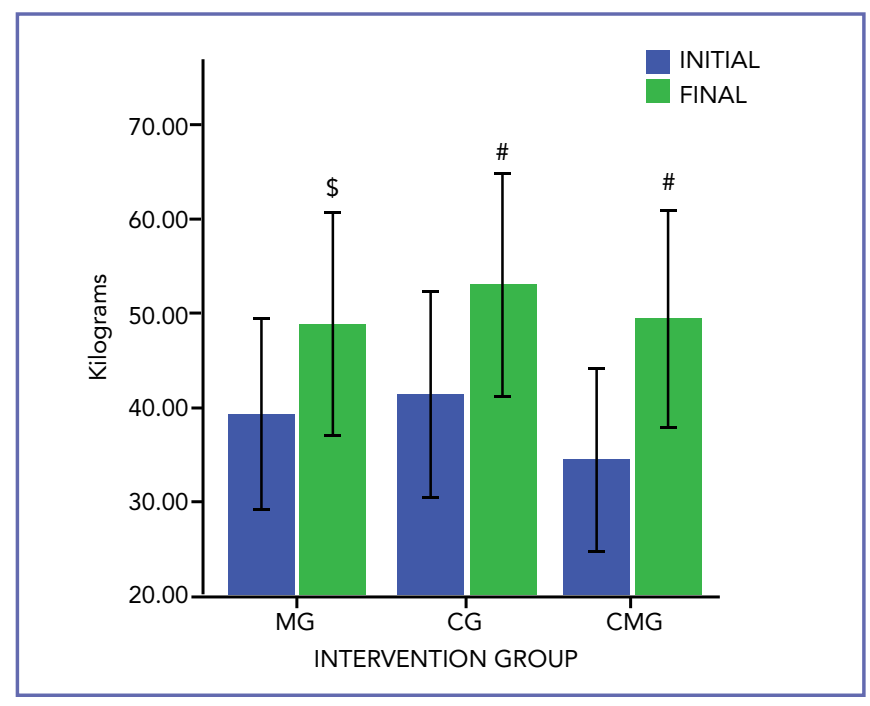

Figure 6. Trunk muscle strength (Maximum Voluntary Isometric Contraction) assessment in the study groups before and after intervention.

GC: Core Group; MG: McKenzie Group; CMG: Core + McKenzie Group.

$\# p<0.001$ compared to baseline in the same group.

$\$ p<0.005$ compared to baseline in the same group. Student's t test.
The ability to perform an isolated contraction of these muscles is the basis of the specific stabilization approach. Inability to master this exercise component prevents patients from progressing on their exercise program (26). The stabilization exercise program applied in this study lasted four weeks. Perhaps many of these patients who participated in the study could have responded more favorably to the program with a longer intervention period. The exercise dosage can significantly affect the treatment of low back pain (25). Miller et al. approached 30 subjects with chronic low back pain using the McKenzie exercises and stabilization exercises for six weeks (9). Both groups improved pain and function after the intervention (10).

Both exercise programs significantly reduced pain intensity after four weeks of intervention. Notwithstanding, it seems to us that the reduction in pain intensity occurs through different mechanisms in the two proposed programs. The positive results associated with the McKenzie approach may relate to the activation of pain gate control mechanisms or to relaxation and/or decompression of neural tissues $(10,16)$. The application of controlled forces to the spine through active exercises or techniques can temporarily reduce pain intensity by altering the fluid dynamics of the injured tissue $(10,16)$. Another theory that may justify this analgesic

Table V. Results of lumbopelvic stability tests in the study groups before and after intervention $(n=69)$.

\begin{tabular}{|c|c|c|c|c|c|c|c|}
\hline \multirow[t]{2}{*}{ Variable } & \multicolumn{2}{|l|}{ CG $(n=23)$} & \multicolumn{2}{|c|}{ MG $(n=23)$} & \multicolumn{3}{|c|}{ CMG (n=23) } \\
\hline & Initial & Final & Initial & Final & Initial & Final & p\$ \\
\hline Single Leg Squat Test & & & & & & & 0.043 \\
\hline Positive & $23(100.0)$ & $9(39.1)$ & $23(100.0)$ & $16(69.6)$ & $20(87.0)$ & $11(47.8)$ & \\
\hline Negative & $0(0.0)$ & $14(60.9)$ & $0(0.0)$ & $7(30.4)$ & $3(13.0)$ & $12(52.2)$ & \\
\hline p\# & 0.000 & & 0.006 & & 0.007 & & \\
\hline Runner Pose Test & & & & & & & 0.003 \\
\hline Positive & $15(65.2)$ & 5 & $16(69.6)$ & 11 & $5(21.7)$ & 1 & \\
\hline Negative & $8(34.8)$ & 18 & $7(30.4)$ & 12 & $18(78.3)$ & 22 & \\
\hline$p \#$ & 0.002 & & 0.025 & & 0.046 & & \\
\hline Prone Instability Test & & & & & & & 0.019 \\
\hline Positive & 20 & 8 & 21 & 15 & 17 & 6 & \\
\hline Negative & 3 & 15 & 2 & 8 & 6 & 17 & \\
\hline$p \#$ & 0.001 & & 0.014 & & 0.002 & & \\
\hline
\end{tabular}

Legend: CG: Core Group; MG: McKenzie Group; CMG: Core + McKenzie Group.

Bold values are statistically significant.

$\$$ Chi-square test.

\#Wilcoxon test. 
Table VI. Results of the posture holding time in the core stabilization tests of the intervention groups $(n=69)$.

\begin{tabular}{|c|c|c|c|c|c|c|c|c|}
\hline Functional Test & Group & $\begin{array}{l}\text { Baseline, } \\
\text { mean } \pm \text { SD }\end{array}$ & $\begin{array}{l}\text { Postintervention, } \\
\text { mean } \pm \text { SD }\end{array}$ & $\mathbf{p}^{\mathrm{a}}$ & $\begin{array}{l}\text { Effect } \\
\text { Size }\end{array}$ & $\begin{array}{l}\text { Score variation within } \\
\text { the group }(95 \% \mathrm{CI})\end{array}$ & $\mathbf{F}$ & $\mathbf{p}^{\mathrm{b}}$ \\
\hline \multirow[t]{2}{*}{ Right side bridge } & CG & $17.13 \pm 17.42$ & $42.30 \pm 35.05$ & 0.000 & -5.33 & $-25.17(-34.97$ to -15.38$)$ & 5.093 & $0.009^{\#}$ \\
\hline & $\mathrm{CMG}$ & $22.17 \pm 15.98$ & $42.08 \pm 25.41$ & 0.001 & -3.83 & $-19.91(-11.32$ to -3.37$)$ & & \\
\hline Left side bridge & CG & $20.34 \pm 16.84$ & $44.30 \pm 24.98$ & 0.000 & -6.59 & $-23.96(-31.50$ to -16.41$)$ & 4.757 & $0.012^{\#}$ \\
\hline \multirow[t]{3}{*}{ Sorenson Test } & CG & $37.70 \pm 29.55$ & $62.96 \pm 37.82$ & 0.001 & -3.90 & $-25.26(-38.69$ to -11.83$)$ & 2.860 & 0.069 \\
\hline & MG & $21.35 \pm 16.84$ & $41.57 \pm 35.04$ & 0.005 & -5.17 & $-20.56(-32.56$ to -9.35$)$ & & \\
\hline & CMG & $30.22 \pm 32.58$ & $68.17 \pm 46.25$ & 0.000 & -6.21 & $-37.95(-50.64$ to -25.27$)$ & & \\
\hline
\end{tabular}

Legend: CG: Core Group; GM: McKenzie Group; CMG: Core + McKenzie Group; CI: Confidence Interval; SD: Standard Deviation.

Bold values are statistically significant

${ }^{\text {a }}$ Intragroup Student's t-test.

${ }^{\mathrm{b}}$ One-Way ANOVA.

" Significant difference between the MG, CG, and CMG.

effect in the McKenzie method is that stimulation of arterial, venous, and lymphatic drainage or mechanoreceptive stimulation with subsequent increase in the afferent pathway to the central nervous system can result in pain modulation and inhibition of the hypertonic muscle (10). Trunk extension programs have improved some of the main stability measures with activation of the local and global muscle system, stabilizing the region around the spine and reducing pain (22).

The literature describes the effects of core stabilization exercises on pain through several mechanisms. These mechanisms include reducing the load and improving the quality of movements after improving trunk muscle coordination (16). Furthermore, stabilization exercises aim to activate mainly the deep muscles, commonly affected by low back pain (16).

Regarding spinal instability, Panjabi $(27,28)$ describes a neutral zone where there is a synergistic interaction between passive and active elements and the neural control of the spine to control the movement between intervertebral segments $(10,29)$. If one of these elements is compromised, for example, by disc degeneration, spinal injury, or muscle dysfunction, an imbalance occurs within the neutral zone resulting in instability of this complex and, consequently, pain $(10,29)$.

Hence, lumbosacral spine instability in the studied population may relate to the presence of an advanced discogenic pathology that was most affected by the extension program. Although McKenzie's conceptual model of pulpal nucleus displacement with repeated movements in the final range so as to reduce abnormal disc pressures remains controver- sial, several studies have demonstrated anterior or posterior nuclear migration away from the compressive forces of flexion or extension movements in the sagittal plane.

Some studies describe core muscle imbalance in patients with low back pain compared to healthy individuals (23). In comparison with general exercises or spinal manipulation, motor control exercises for patients with low back pain improve the size and recruitment of deep spinal muscles, including $\operatorname{Tr} A$, improving pain and short-term function (24). De Georgio, Padulo and Kuvacic (30) reported that people who develop chronic pain in the absence of other associated pathologies, as in the case of chronic non-specific low back pain, may be predisposed to avoid some gestures, movements, daily routines and/or sports activities with the intention to decrease the pain. This can lead to a significant reduction in the level of physical activities, that is, a "disuse syndrome", which increases the perception of stiffness and back pain. All of these aspects can lead to the development of a vicious circle that results in the worsening of the patient's quality of life.

The proposed mechanism of action for motor control exercises involves better coordination of deep spinal and trunk muscles, such as TrA and Internal Oblique (IO) muscles (24). There is a better ratio between $\operatorname{TrA}$ and IO muscle activation and rectus abdominis improvement in individuals treated with core stabilization exercises (30). These exercises increase the activation of segmental muscles, reducing pain in individuals with chronic nonspecific low back pain (30).

Hosseinifar et al. demonstrated that stabilization exercises were more effective than McKenzie exercises in increas- 
ing the resting thickness of the left and right $\operatorname{Tr} A$ muscles and the multifidus muscle (15). These findings corroborate those of the present study, where the CG had a greater activation of these muscle groups, as shown by the Lateral Bridge, Single Leg Squat, and Prone Instability tests.

One of the most widely validated outcome measures for low back pain is the Roland-Morris Disability Questionnaire (RMDQ) (23). The present study showed a significant reduction in the RMDQ score in all intervention groups, with no differences between them. The possible explanations for these findings are the improvement of trunk muscle activity and the increase in the range of lumbar motion, leading to less disability and functional recovery. According to the fear-avoidance model, people's fear of low back pain aggravates pain, leading to chronic pain and, ultimately, permanent functional disability (22).

In the treatment of low back pain using McKenzie and, especially, stabilization exercises, focusing on the preferred direction of the TrA and multifidus muscles, respectively, relieves pain, which helps patients to recover physically and psychologically (22). Exercises combat chronic pain and functional disability by conquering fear and, subsequently, preventing pain in patients (22). Thus, patients report reduced pain and disability scores after interventions (22). The specificity of the exercises helps in managing pain that stems from tense muscles and therefore facilitates healing (22). In other words, exercises help patients to cope with their pain, leading to recovery (22). Chopani et al. (32) approached 24 subjects with spondylolisthesis, randomly divided into a group of stabilization exercises or a group of general exercises. The authors observed that pain and disability improved in both groups, but the variables of postural stability were not changed in different directions in the stabilization group. This result can demonstrate that several exercise methods can offer benefits on low back pain and disability. Notarnicola et al. (33) approached 60 people with chronic non-specific low back pain divided into a group receiving the Pilates method and a control group. Participants in the Pilates group received an hour of daily exercise, five times a week for six months. The Pilates group demonstrated an important improvement in pain, disability, and physical and psychological health perception.

Results suggest that both exercise programs improve pain and functional disability through their own and distinct mechanisms. The choice of one or the other seems to be an important contributor to the previously considered clinical outcomes (26). Patients and clinicians may use either approach to improve long-term clinical outcomes. However, this choice must consider both the clinical experience with the technique and the response of each patient. In addition, it is important to emphasize the importance of exercise as a routine physical activity program in the prevention of low back pain originating from a degenerative process of the intervertebral disc.

\section{Study limitations}

This research has limitations such as the lack of evaluation and control of analgesic intake by patients during the study period. Another limitation is the lack of a follow-up to observe the long-term effects of the exercise programs administered. The results are further limited by a small sample size that limits statistical power. In addition, the study was pragmatic, without a true control group (non-intervention). Future studies are necessary to include such variables so as to correlate and enhance the results of this research.

\section{CONCLUSIONS}

The results of this study suggest that McKenzie and core stabilization exercises, either alone or in association, are effective in treating low back pain due to Disc Protrusion (DP). In particular, core exercises enhanced lumbopelvic complex stabilization and functional capacity according to the domains of the SF-36 quality of life questionnaire.

\section{ACKNOWLEDGEMENTS}

We thank the Group of Studies and Research in Sports and Orthopedic Trauma Rehabilitation, Physiotherapy Course, Lutheran University of Brazil, Torres/RS/Brazil.

\section{CONFLICT OF INTERESTS}

The authors declare that they have no conflict of interests.

\section{REFERENCES}

1. Zhu H, Zhou XZ, Cheng MH, et al. The efficacy of coblation nucleoplasty for protrusion of lumbar intervertebral disc at a two-year follow-up. Int Orthop 2011;35(11):1677-82.

2. Fardon DF, Williams AL, Dohring EJ, et al. Lumbar disc nomenclature: version 2.0 Recommendations of the combined task forces of the North American Spine Society, the American Society of Spine Radiology and the American Society of Neuroradiology. Spine J 2014;14(11):2525-45.

3. Li Y, Fredrickson V, Resnick DK. How Should We Grade Lumbar Disc Herniation and Nerve Root Compression? A Systematic Review. Clin Orthop Relat Res 2015;473(6):1896-902.

4. Song H, Sheng H, Xu W. A case-control study on the treatment of protrusion of lumbar intervertebral disc through PELD and MED. Exp Ther Med 2017;14(4):3708-12. 
5. Shao J, Yu M, Jiang L, et al. Sequencing and bioinformatics analysis of the differentially expressed genes in herniated discs with or without calcification. Int J Mol Med 2017;39(1):81-90.

6. Plaza-Manzamo G, Cancela-Cilleruelo I, Fernández-de-laPenãs C, et al. Effects of Adding a Neurodynamic Mobilization to Motor Control Training in Patients with Lumbar Radiculopathy due to Disc Herniation: A Randomized Clinical Trial. Am J Phys Med Rehab 2019;99(2):124-132.

7. Pourahmadi MR, Taghipour M, Takamjani, IE, et al. Motor control exercise for symptomatic lumbar disc herniation: protocol for a systematic review and meta-analysis. BMJ Open 2016;6(9):24-6.

8. Ghosh S, Datta S, Nayak S, Chaudhuri A, Dhanasekaran P. Comparative study of muscle strengthening exercises for treatment of chronic low backache. Med J DY Patil Univ 2014;7:443-6.

9. Mbada CE, Ayanniyi O, Ogunlade SO, et al. Influence of Mckenzie protocol and two modes of endurance exercises on health-related quality of life of patients with long-term mechanical low-back pain. Pan Afr Med J 2014;17(1):1-7.

10. Miller ER, Schenck RJ, Karnes JL, Rousselle JG. A Comparison of the McKenzie Approach to a Specific Spine Stabilization Program for Chronic Low Back Pain. J Man Manip Ther 2005;13(2):103-12.

11. Hasanpour-Dehkordi A, Dehghani A, Solati K. A Comparison of the Effects of Pilates and McKenzie Training on Pain and General Health in Men with Chronic Low Back Pain: A Randomized Trial. Indian J Palliat Care 2017;23(1):36-40.

12. Dunsford A, Kumar S, Clarke S. Integrating evidence into practice: use of McKenzie-based treatment for mechanical low back pain. J Multidiscip Healthc 2011;4:393-402.

13. Garcia AN, Gondo FLB, Costa RA, et al. Effectiveness of the back school and Mckenzie techniques in patients with chronic non-specific low back pain: a protocol of a randomised controlled trial. BMC Musculoskelet Disord 2011;12:1-7.

14. Elenburg JL, Foley BS, Roberts K, Bayliss AJ. Utilization of mechanical diagnosis and therapy (MDT) for the treatment of lumbar pain in the presence of known lumbar transverse process fractures: a case study. J Man Manip Ther 2016;24(2):74-9.

15. Zheng Y, Ke S, Lin C, et al. Effect of Core Stability Training Monitored by Rehabilitative Ultrasound Image and Surface Electromyogram in Local Core Muscles of Healthy People. Pain Res Manag 2019;23:1-8.

16. Hosseinifar M, Akbari M, Behtash H, et al. The Effects of Stabilization and Mckenzie Exercises on Transverse Abdominis and Multifidus Muscle Thickness, Pain, and Disability: A Randomized Controlled Trial in NonSpecific Chronic Low Back Pain. J Phys Ther Sci 2013;25(12):1541-45.

17. Paungmali A, Henry LJ, Sitilertpisan P, et al. Improvements in tissue blood flow and lumbopelvic stability after lumbopelvic core stabilization training in patients with chronic non-specific low back pain. J Phys Ther Sci 2016;28(2):635- 40.

18. Hoppes CW, Sperier AD, Hopkins CF, et al. The Efficacy of an Eight-week Core Stabilization Program on Core Muscle Function and Endurance: A randomized trial. Int J Sports Phys Ther 2016;11(4):507-15.

19. Akhtar MW, Karimi H, Gilani AS. Effectiveness of core stabilization exercises and routine exercise therapy in management of pain in chronic non-specific low back pain: A randomized controlled clinical trial. Pak J Med Sci 2017;33(4):1002-6.
20. Ki C, Heo M, Kim HY, Kim EJ. The effects of forced breathing exercise on the lumbar stabilization in chronic low back pain patients. J Phys Ther Sci 2016;28(12):3380-83.

21. Padulo J, Oliva F, Frizziero A, Maffuli N, Muscles, Ligaments and Tendons Journal - Basic principles and recommendations in clinical and field Science Research: 2018 update. MLTJ 2018;8(3):305-7.

22. Moon HJ, Choi KH, Kim DH. Effect of Lumbar Stabilization and Dynamic Lumbar Strengthening Exercises in Patients with Chronic Low Back Pain. Ann Rehabil Med 2013;37(1):110-17.

23. Alhakami AN, Davis S, Qasheesh M, Shaphe A, Chaal A. Effects of McKenzie and stabilization exercises in reducing pain intensity and functional disability in individuals with nonspecific chronic low back pain: a systematic review. J Phys Ther Sci 2019;31:590-97.

24. Shansi M, Mirzaei M, HamediRad M. Comparison of muscle activation imbalance following core stability or general exercises in nonspecific low back pain: a quasi-randomized controlled trial. BMC Sports Sci Med 2020;12(24):1-9.

25. Halliday MH, Papas E, Hancock MJ, Clare HA, Pinto RZ, Robertson G, Ferreira PH. A Randomized Controlled Trial Comparing the McKenzie Method to Motor Control Exercises in People With Chronic Low Back Pain and a Directional Preference. J Orthop Sports Phys Ther 2016;46(7):514-22.

26. Halliday MH, Papas E, Hancock MJ, Clare HA, Pinto RZ, Robertson G, Ferreira PH. A randomized clinical trial comparing the McKenzie method and motor control exercises in people with chronic low back pain and a directional preference: 1-year follow-up. Physiotherapy 2019;105:442-45.

27. O'Sullivan PB, Phyty GD, Twomey LT, Allison GT. Evaluation of specific spine stabilizing exercise in the treatment of chronic low back pain with a radiographic diagnosis of spondylolisthesis or spondylolysis. Spine 1997;22:2959-2967.

28. Panjabi MM. The stabilizing system of the spine. Part i. function, dysfunction, adaptation, and enhancement. J Spinal Disord 1992;5:383-89.

29. Panjabi MM. The stabilizing system of the spine. Part ii. neutral zone andinstability hypothesis. J Spinal Disord 1992;5:390-97.

30. Tang S, Qian X, Zhang Y, Liu Y. Treating low back pain resulted from lumbar degenerative instability using Chinese Tuina combined with core stability exercises: A randomized controlled trial. Complement Ther Med 2016;25:45-50.

31. De Georgio A, Padulo J, Kuvacic G. Effectiveness of yoga combined with back school program on anxiety, kinesiophobia and pain in people with non-specific chronic low back pain: a prospective randomized trial. MLTJ 2018;8(1):104-12.

32. Luz RD, Santos M, Evaldt A, Matos LS, Daitx RB, Dohnert MB. Neuromuscular electrical stimulation associated with core stability exercises in nonspecific postural low back pain: a randomized clinical trial. MLTJ 2019;9(3):446-56.

33. Chopani R, Ghaderi F, Salahzadeh Z, Adigozali H, Sivaki HN, Majd EM, Azghani MR, Soltani A, Jafarabadi MA. The Effect of Segmental Stabilization Exercises on Pain, Disability and Static Postural Stability in Patients with Spondylolisthesis: A Double Blinded Pilot Randomized Controlled Trial. MLTJ 2019;9(4):615-26.

34. Notarnicola A, Fischetti F, Maccagnano G, Comes R, Tafuri S, Moretti B. Daily Pilates exercise or inactivity for patients with low back pain: a clinical prospective observational study. Eur J Phys Rehabil Med 2014;50(1):59-66. 